\title{
Correlation of Pain Scores, Analgesic Use, and Beck Anxiety Inventory Scores During Hospitalization in Lower Extremity Amputees
}

\author{
Cathy D. Trame ${ }^{*}$, , Erin Greene ${ }^{2}$, Gail Moddeman ${ }^{3}$, Branyan A. Booth ${ }^{4}$, Emmanuel K. Konstantakos ${ }^{4}$, \\ Stephen Parada ${ }^{5}$, Karl Siebuhr ${ }^{4}$ and Richard T. Laughlin ${ }^{4}$ \\ ${ }^{I}$ Miami Valley Hospital, Pain Center, 30 East Apple Street, Suite 5250, Dayton, Ohio 45409, USA \\ ${ }^{2}$ Good Samaritan Hospital, 3084 Village Court, Beavercreek, Ohio 45432, USA \\ ${ }^{3}$ Miami Valley College of Nursing and Health, 3640 Colonel Glenn Highway, Dayton, Ohio 45435, USA \\ ${ }^{4}$ Wright State University Boonshoft School of Medicine, Department of Orthopaedic Surgery \& Sports Medicine, Miami \\ Valley Hospital, 30 East Apple Street, Suite 2200, Dayton, Ohio 45409, USA \\ ${ }^{5}$ Madigan Army Medical Center, 9040A Fitzsimmons Drive, Tacoma, Washington 98431, USA
}

\begin{abstract}
Post amputation pain can be debilitating for patients and families. Chronic pain is a common phenomenon after lower extremity amputation, occurring in up to $80 \%$ of this population. The purpose of this pilot study was to correlate post amputation pain scores to opioid analgesic consumption and Beck Anxiety Inventory (BAI) scores. Twenty-three patients with lower extremity amputation at an 827 -bed acute care inner-city hospital were surveyed pre-operatively and post-operatively to determine if there was a significant correlation between anxiety and pain. A numeric scale was utilized by patients to rate their pain level, while the BAI was utilized to measure their anxiety levels.

A significant correlation was found between the pre-operative BAI levels and the BAI levels identified at time of discharge. Patients were found to have a higher than normal level of anxiety pre-operatively. No significant correlations were found between anxiety and pain.
\end{abstract}

Keywords: Amputation, Pain, Beck Anxiety Inventory, Anxiety, Phantom Limb.

\section{PURPOSE}

The purpose of the study was to determine if anxiety scores significantly correlated with pain scores and opioid analgesic use in surgical patients having lower extremity amputations.

\section{BACKGROUND}

Chronic pain and functional outcomes are important long term issues that lower extremity amputees face. It is well known that emotions such as shock, denial, grief, are common amongst lower extremity amputees. It is when these emotions are met with inadequate coping strategies that depressive symptoms may occur. The affective distress coupled with chronic pain may adversely affect the patient and prevent them from having a successful outcome. We had hypothesized that if the patient's pain and their psychological well-being is managed effectively, then the patient will have a successful long term functional outcome.

Amputations of all types have been shown to predispose the patient to the likelihood of chronic limb pain or chronic phantom pain [1-4]. A variety of studies have shown that chronic pain develops very early in the acute phase of recov-

*Address correspondence to this author at the Miami Valley Hospital, Pain Center, 30 East Apple Street, Suite 5250, Dayton, Ohio 45409, USA;

Tel: 937-208-5127; E-mail: cdtrame@mvh.org ery $[5,6]$. Therefore, immediate aggressive management of amputation pain is crucial.

Dijkstra and associates found the prevalence of phantom pain to be $80 \%$ in 434 lower limb amputees [7]. Three factors which significantly increased the risk for phantom pain included: bilateral amputation; lower limb amputation (particularly above the knee), and increased age.

Gallagher et al. showed that there was a $67 \%$ incidence of phantom pain in patients six months or greater post amputation $[2,8]$. In their report, continuous analgesia with local anesthetic through nerve sheath catheters was utilized intraoperatively on all patients. Pain score on a visual analog scale equal to or greater than three was utilized to determine the presence of phantom pain. Although the overall incidence of phantom pain was reduced with their use of this analgesic technique, the high percentage of affected patients remains disturbing.

A study of 104 lower extremity amputation patients revealed $48.1 \%$ with residual limb pain (pain in the remaining stump), while $69.2 \%$ experienced phantom limb pain [9]. Interestingly, those with residual limb pain reported a "greater interference in their daily lifestyle" with increased pain intensity, as compared to those with phantom limb pain. Another study of 437 patients with lower extremity amputations revealed that phantom pain significantly interfered with quality of life, as compared to amputees without pain. Similarly, a cross sectional study of 914 patients revealed a $95 \%$ 
incidence of amputation-related pain with phantom pain being reported most often (79.9\%), followed by residual limb pain $(67.7 \%)$ [10].

In a study of 52 patients post-amputation, Richardson et al. found an incidence of $78.8 \%$ of phantom limb pain and a $51.2 \%$ incidence of stump pain [11]. They also noted that phantom sensations were universal among a homogenous group of amputees with peripheral vascular disease although the impact of pain on functional status was not measured.

Desmond and MacLachlan found that $87 \%$ of patients surveyed $(\mathrm{N}=582)$ had either phantom limb or residual limb pain [12]. A correlation was noted between the patients with chronic limb pain and higher affective distress scores. Price also found significant correlation $(p<0.05)$ between physical, social, and emotional satisfaction and phantom pain [13]. Ephraim et al. showed depressive symptoms were a significant predictor of the level of pain intensity and "bothersomeness" [10]. This suggests that patients who have experienced a lower limb amputation and have chronic limb pain, may benefit from therapeutic interventions that would treat the patient's emotional and social well being.

The long term effects of pain and disability associated with lower limb amputation certainly make this topic important and timely with recent combat-related injuries. In a retrospective study of 255 patients with six months or greater post lower limb amputation, phantom limb pain was reported by $72 \%$ of respondents, while $74 \%$ reported residual limb pain [14]. Although most respondents with pain did not find the pain disabling (47\%), $28 \%$ of the group found the pain to be of "high intensity". An association between anxiety and postoperative pain scores may provide clues to more comprehensive treatment of the amputation patient.

\section{METHODS}

\section{Setting}

This pilot study was conducted in an 827 bed acute care Level I Trauma Center located in the MidWest. Institutional Review Board approval was obtained prior to initiation of the study.

\section{Sample}

In accordance with the institution's protocol, patients agreeing to participate via written informed consent were included in the study. Potential participants were identified by review of the daily surgery schedule. English speaking men and women who were eighteen years or older, and undergoing lower extremity amputation (LEA) were eligible for this study. No patients in the study received pre-operative analgesia. LEA was defined as any of the following procedures: above-knee amputation (AKA), below-knee amputation (BKA), or revision surgeries within any of those classes.

\section{Procedures}

Data were collected for this study prospectively, patients were assessed pre-operatively, immediately post-operatively, and ending at time of discharge. On postoperative day one, defined as the first 24 hours after surgery, commencing in the PACU, data related to the mean pain score pre-treatment, pain location, mean pain score post-treatment, and pharmacologic use were collected. Numeric pain scores were re- corded from nursing notes. Pain location consisted of residual limb pain, phantom pain or other pain. Pharmacologic use was defined as opioid or adjuvant drug therapy that could influence pain outcomes. Adjuvant medications included anxiolytics and agents used to treat neuropathic pain (anticonvulsants, tricyclic antidepressants, skeletal muscle relaxants, and alpha-2 agonists). Post-operative pharmacologic use was the total opioid use within the 24 hour period beginning with the onset of surgery, including the amount of opiate use intraoperatively by anesthesia. Day of discharge pharmacologic use was the total opioid use during the 24 hour period prior to discharge. All opioid pharmacologic interventions were calculated into morphine equivalents for the 24 hour period using the hospital's current protocol.

\section{Exclusion Criteria}

Exclusions from the study included:

- $\quad$ Non-verbal patients or non-communicative patients

- $\quad$ Patients with psychiatric disorders

- Patients with dementia or confusion

- $\quad$ Non English-speaking patients

- Patients who were not able to be interviewed and have information gathered prior to their operation due to time constraints

- $\quad$ Patients who refused to participate in the study

\section{Description of the Tools}

The Beck Anxiety Inventory (BAI) is a self report tool that was developed to measure anxiety in adult psychiatric outpatients [14]. The BAI has become a frequently used tool to measure anxiety in many different patient populations because it is cost effective, efficient, user-friendly, and is easy to interpret and complete. The BAI contains 21 items that are designed to assess severity of anxiety. The responses to each item range from; not at all, mildly (it did not bother me much.), moderately (it was very unpleasant, but I could stand it) to severely (I could barely stand it). The ranges of scores are from 0-63, with the higher the score correlating to the higher level of anxiety. The internal consistency and testretest reliability estimates of the BAI are reported to be .92 and .75 respectively [15]. In a recent study regarding general reliability, good internal consistency with co-efficient alpha score of at least a mean of 0.83 or better was obtained [16]. The BAI when compared to other anxiety assessment tools has shown to have a convergent validity correlation range of .47 to .81 . BAI literature does support that gender, diagnostic classification, the participant's primary language, and sample size all influence reliability ratings. Race and ethnicity are additional variables that may affect BAI reliability however there is currently insufficient evidence to support this claim.

The numeric pain intensity scale is a self report tool used by the patients to capture their perception of pain. The pain scale ranges from 0 through 10 . "Zero" meaning no pain to " 10 " meaning the worst pain imaginable. The patients were asked pre operatively, upon discharge, and periodically through their hospital stay to rate their pain using the numeric pain intensity scale. To ensure reliability and validity throughout the study the numeric rating scale (NRS) was the 
only pain scale used. In order to assure consistency all patients received brief instruction pre-operatively and postoperatively on how to use the NRS. The NRS has been reported in previous studies to be reliable and valid when used consistently, as well as easy to understand and administer [17].

The data collection tool was designed by the investigators to capture a variety of variables over a ten year period (Appendix A). The tool was completed by an orthopedic physician research fellow or the principal investigator (clinical nurse specialist) for the pilot study population.

\section{RESULTS}

\section{Demographics of Study Population}

The patients enrolled in the pilot study included a total of 23 patients. Ten patients were female and 13 patients were male. The age range for the study was 42 to 99 years, with a mean age of 63.61 years and a median age of 63 (standard deviation of 12.8). The majority of the patients had vascular insufficiency $(\mathrm{n}=14)$, while the remaining 6 were diabetic, and 3 had "other" physiologic causes for amputation. Eleven of the patients had below the knee amputations ( 5 were revisions), and twelve of the patients had above the knee amputations ( 2 were revisions). The length of stay ranged from 1 day to 14 days with a mean of 5.39 days, and median of 5 days, and a standard deviation of 3.4 days. Pain ratings preoperatively, 24 hours after the operation and day of discharge are reported in Table 1. BAI scores pre-operatively and day of discharge are also reported in Table $\mathbf{1 .}$
Post-op BAI

$$
\begin{aligned}
& r=.663, \underline{\text { significant }}(\mathrm{p}=.001) \\
& \mathrm{r}=-.079, \text { not significant }(\mathrm{p}=.719) \\
& \mathrm{r}=-.115, \text { not significant }(\mathrm{p}=.602)
\end{aligned}
$$

Opioid doses post-op

Opioid doses at discharge

The only correlation which was statistically different from zero was the one between pre-operative and postoperative BAI scores. Assuming that the test-retest correlation has been significant in other settings, it was not surprising that there was a positive relationship between the two, that is, elevations in pre-op anxiety were reflected by elevations in post-op anxiety. One might presume that the BAI elevation was not affected by the surgical procedure since the elevations did not reduce significantly post-operatively, however, correlation between pain and BAI at both times was not statistically significant.

There were two correlations which were negative, that is, as BAI score decreased, opioid administration increased, however both of these correlations were so small that they could just as easily be said to be zero. The possibility that people with lower anxiety scores require more opioid medication cannot be concluded and warrants further study.

The patients also were divided into two groups: those who had spinal administration of analgesic medication $(\mathrm{n}=6)$, and those who did not $(n=17)$. The correlation between the preoperative BAI score and the amount of postoperative opioid medication used for both groups was not significant. For the six individuals who did have an epidural, the correlation was .131 , which is not significant $(\mathrm{p}=.804)$. For the 17 who did not have an epidural, the correlation was -.135,

Table 1. Age, BAI pre-op, BAI Discharge, Length of Stay (LOS), and Pain Ratings of Study Sample

\begin{tabular}{|l||c|c|c|c|c|c|c|}
\hline & Age & BAI Pre-op & BAI_DC & Length of Stay & Pain: Pre-op & Pain: Post-op & Pain_DC \\
\hline \hline $\mathbf{n}$ & 23 & 23 & 23 & 23 & 23 & 23 \\
\hline Mean & 63.61 & 9.96 & 7.39 & 5.39 & 5.217 & 5.507 \\
\hline Median & 63 & 6 & 6 & 5 & 7 & 5.2 & 5.4 \\
\hline Std. Deviation & 12.809 & 9.934 & 7.247 & 3.448 & 4.0922 & 2.2543 \\
\hline Minimum & 42 & 0 & 0 & 1 & 0 & 1.4 & 0 \\
\hline Maximum & 99 & 32 & 24 & 14 & 10 & 8.4 \\
\hline
\end{tabular}

\section{Data Analysis}

Data was analyzed using descriptive statistics to obtain medians, means, standard deviation and range. Descriptive statistics and correlations between variables were measured using SPSS with the level of significance equaling $\leq 0.05$. The first research question was, "What was the relationship between the pre-operative and post-operative BAI score and the numeric pain score?" The correlations between the preoperative BAI, numeric pain scores, and opioid use were as follows:

Pre-op BAI and:

$$
\begin{array}{ll}
\text { Pre-op pain } & \mathrm{r}=.069, \text { not significant }(\mathrm{p}=.754) \\
\text { Post-op pain } & \mathrm{r}=.104, \text { not significant }(\mathrm{p}=.638) \\
\text { Pain at discharge } & \mathrm{r}=-.076, \text { not significant }(\mathrm{p}=.732)
\end{array}
$$

which also is not significant $(\mathrm{p}=.604)$. There was no significant correlation between the post-operative BAI levels and pain scores and post-operative opioid use.

\section{DISCUSSION}

The study's main research question was "Is there a relationship between, the pre-operative and post-operative BAI score and the numeric pain score?" When a statistical analysis was done to calculate the correlation between the pre-op BAI scores and other variables it was found that only one significant correlation existed. The significant correlation showed a positive relationship between pre-op and post-op BAI scores. No significant correlation could be found between BAI scores, pain score, and analgesic use.

A major limitation of this pilot study was its small sample size $(n=23)$. Data was collected for one year (approved time for data collection). Fifty percent of all amputations with in- 
clusive criteria during the one year study period, participated in the study. The sample size may have contributed to the low statistical power and the inability to detect significant correlations between anxiety and pain scores. The study also only examined acute pain and anxiety while the patient was hospitalized. Providing an analysis of acute and long term pain and anxiety data may have proven to be more insightful and descriptive. Furthermore, BAI scores were collected during presumably stressful times, pre-operatively and on the day of discharge. One may hypothesize that the times that the BAI were administered could contribute to the higher than accepted BAI scores (mean of 9.96), accepted level of minimal anxiety is seven [15]. An additional limitation of the BAI tool included variables that may be present due to the physiologic symptoms of vascular disease versus symptoms induced by anxiety; that is numbness or tingling, wobbliness in legs, and unsteady.

Pre-operative and post-operative pain scores in the lower extremity were not statistically significant from each other. We feel that this may attributed to the fact that most patients who underwent a BKA were experiencing pain before their amputation and that this did not subside regardless of the level of anxiety produced. Hence, reducing pre-operative anxiety levels did not cause sufficient reductions in post-operative pain and that reducing anxiety may only temporarily ameliorate post-operative pain.

The amputations were not isolated to one specific type; that is, AKA and BKA. The extent of the amputation may affect anxiety levels of the patient and thereby be a limitation of this study. Other confounding variables such as the patient's diagnoses and co-morbidities, patient's surgeon and surgical technique, were not controlled when assessing anxiety or pain.

These findings suggest that anxiety is not a predictor of pain scores, and treatment of anxiety may not reduce numeric pain scores. These findings are consistent with other research that has been done by Carrington et al. [18] \& Marshal et al. [19]. Due to the construct and limitations of this study, further research needs to be done prior to dismissing the relationship between pain and anxiety in lower extremity amputations. Research using a qualitative method may provide more insightful data related to pain and anxiety. A larger sample would allow for further sorting of variables to determine the significance of each variable.

A national survey conducted by Darnall et al. examined depressive symptoms and mental health service utilization in persons with limb loss $(n=914)$ [20]. The study found that risk factors for emotional depression included being divorced or separated, living at or close to the poverty level, having comorbid medical conditions, and having chronic pain. Chronic pain was identified as residual limb pain, phantom pain or back pain.

The problem of chronic pain and lack of psychological well-being may be further magnified in specific amputee populations. In a study by Gallagher and MacLachlan $(n=44)$ it was found that those individuals who experience an amputation as a result of a trauma had greater limb pain and more maladaptive coping strategies than those individuals that had an amputation as a result of a disease process [21]. In their report, patients with a below the knee amputation had less pain than those who had an above the knee amputation.

Desmond and MacLachlan noted increased anxiety levels in an inpatient amputee population [22]. However, no statistical difference was found when the postoperative amputees' anxiety levels were compared to other postoperative populations. Therefore, anxiety may not be a risk factor that can be used to predict the development of chronic pain or affective distress disorders.

Studies to evaluate the correlation between anxiety and surgical pain have yielded mixed results. A comprehensive review of literature regarding the predictability of postoperative pain based on pre-operative anxiety showed inconsistencies in the literature, however the majority of studies revealed a positive correlation between pain and anxiety [23]. More recent studies have also shown inconsistent results. Ciccozzi et al. found that lorazepam reduced perioperative anxiety, decreased postoperative pain scores, and reduced analgesic use in abdominal hysterectomy patients [24]. A study regarding anxiety in patients undergoing coronary bypass surgery revealed low to moderate anxiety levels before and after surgery using the Hospital Anxiety and Depression Scale [25]. Although pain scores and analgesic use were not measured, some predictors of anxiety were identified including "being in pain or discomfort". In an attempt to predict treatment outcomes based on symptoms of distress, Edwards et al. evaluated patients with sciatica [26]. Their findings suggested that depression and anxiety positively correlated with self-reported pain and disability in patients with disc herniation causing sciatica.

Despite the lack of correlation between pain and anxiety in this pilot study, changes in standards of care for amputees can be made. The research findings did reveal pain scores (a mean of 5 both pre and post operatively) of great concern. These pain scores were not ignored and proved to be the catalyst for changing the care for amputation patients within the institution studied. This forces one to examine current pain regimens, and encourages healthcare providers to look for more effective methods of pain management. Such methods may include utilizing femoral and/or popliteal nerve blocks, continuous local anesthestic infusions, adjuvant medications, and/or exploring alternative surgical techniques.

\section{CONCLUSIONS AND RECOMMENDATIONS}

Certainly the discovery of elevated numeric pain scores along with higher than normal anxiety levels warranted changes in pharmacologic practices for the lower extremity amputation population. Despite the insignificant correlation between pain and anxiety found in this study, the management of both symptoms could be improved. Further research is warranted to determine if the small sample in this pilot study influenced the findings. Additional studies to determine best analgesic practices are needed to reduce the high incidence of chronic pain associated with amputations.

\section{ACKNOWLEDGEMENTS}

The authors express appreciation to Miami Valley Hospital Foundation for providing a grant to purchase the Beck Anxiety Inventories utilized in the study. Appreciation is also extended to the Wright State University Statistical Consulting Center for data analysis. 


\section{APPENDIX A}

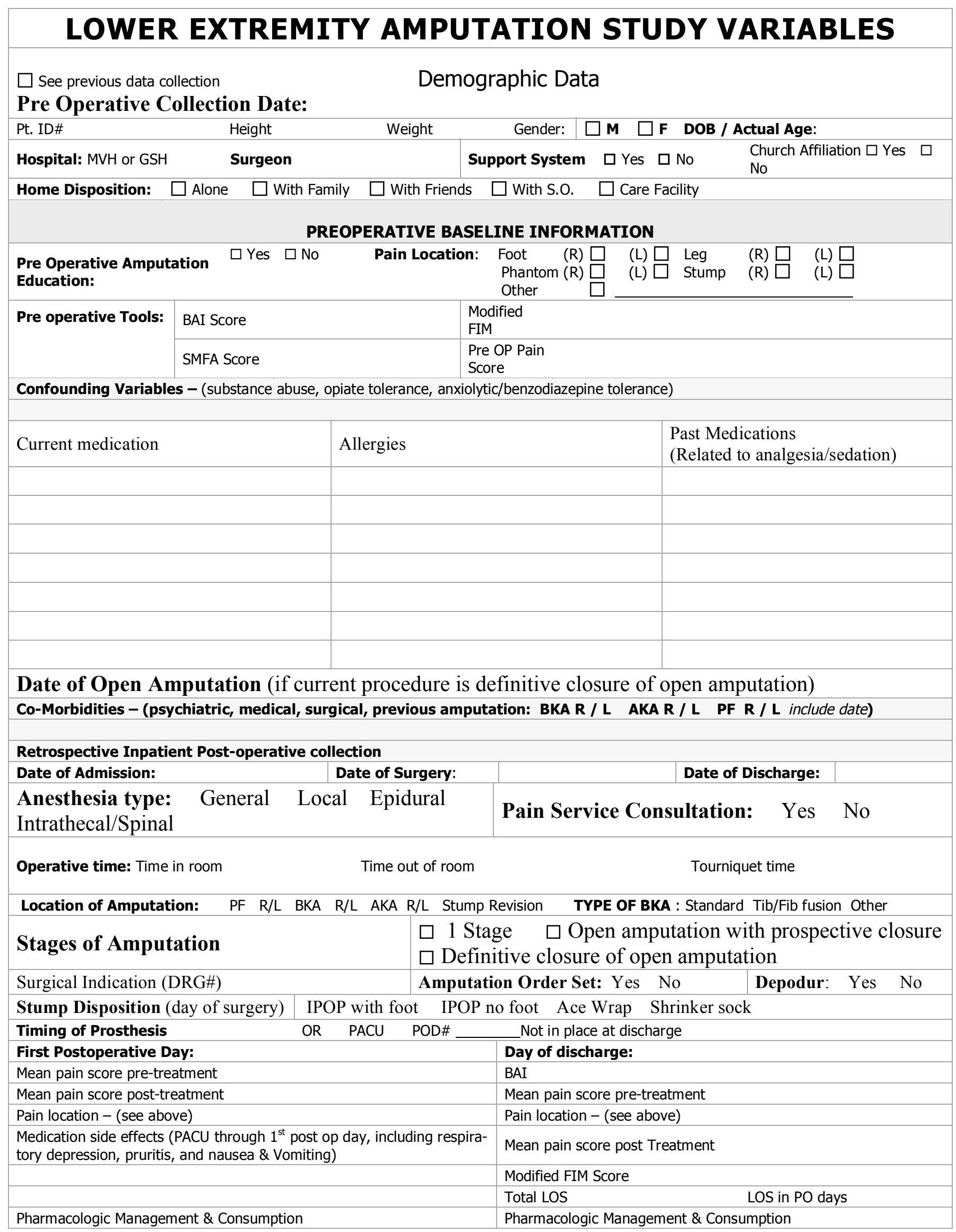




\section{REFERENCES}

[1] Rudy T, Lieber S, Boston J, Gourley L, Baysal E. Psychosocial predictors of physical performance in disabled individuals with chronic pain. Clin J Pain 2003; 19(1): 18-30.

[2] Morey T, Giannoni J, Duncan E, Scarborough M, Enneking F. Nerve sheath catheter analgesia after amputation. Clin Orthop 2002; 397: 281-9.

[3] Middleton CO. The causes and treatments of phantom limb pain. Nursing Times 2003; 99(35): 30-3.

[4] Flor H. Remapping somatosensory cortex after injury. Adv Neurol 2003; 93: 195-204.

[5] Flor $\mathrm{H}$. The modification of cortical reorganization and chronic pain by sensory feedback. Appl Psychophysiol Biofeedback 2002; 27(3): 215-27.

[6] Bloomquist T. Amputation and phantom limb pain: a painprevention model. AANA J 2001; 69(3): 211-7.

[7] Dijkstra P, Geertzen J, Stewart R, van der Schans C. Phantom pain and risk factors: a multivariate analysis. J Pain Symptom Manage 2002; 24(6): 578-85.

[8] Gallagher P, Allen D, Maclachlan M. Phantom limb pain and residual limb pain following lower limb amputation: a descriptive analysis. Disabil Rehabil 2001; 23(12): 522-30.

[9] Van der Schaus C, Geertzen J, Schoppen T, Dijkstra P. Phantom pain and health-related quality of life in lower limb amputees. J Pain Symptom Manage 2002; 24(4): 429-36.

[10] Ephraim PL, Wegener ST, Mackenzie EJ, Dillingham TR, Pezzin LE. Phantom pain, residual limb pain, and back pain in amputees: results of a national survey. Arch Phys Med Rehabil 2005; 86(10): 1910-19.

[11] Richardson C, Glenn S, Nurmikko T, Horgan M. Incidence of phantom phenomena including phantom limb pain 6 months after major lower limb amputation in patients with peripheral vascular disease. Clin J Pain 2006; 2(45): 353-8.

[12] Desmond D, MacLachlan M. Affective distress and amputationrelated pain among older men with long-term, traumatic limb amputations. J Pain Symptom Manage 2006; 31(4): 362-68.

[13] Price J. Exploring the phantom phenomenon from a psychophysiological perspective. J Prosthet Orthot 2005; 17(3): 8795.
[14] Ehde D, Czerniecki J, Smith D, et al. Chronic phantom sensations, phantom pain, residual limb pain, and other regional pain after lower limb amputation. Arch Phys Med Rehabil 2000; 81: 1039-44.

[15] Beck A, Steer R. Manual for the Beck Anxiety Inventory. San Antonio, Texas, Psychological Corporation, 1990.

[16] Ayala RJ, Vonderharr-Carlson DJ, Doyoung K. Assessing the reliability of the Beck Anxiety Inventory scores. Educ Psychol Meas 2005; 5(65): 742-56.

[17] McCaffery M, Pasero C. Pain Clinical Manual, $2^{\text {nd }}$ ed. St., Louis, Mosby, 1999.

[18] Carrington A, Mawdsley S, Morley M, Kincey J, Boulton A. Psychological status of diabetic people with or without lower limb amputation. Diabetes Res Clin Pract 1996; 32(1-2): 19-25.

[19] Marshall M, Helmes E, Deathe AB. A comparision of psychosocial functioning and personality in amputee and chronic pain populations. Clin J Pain 1992; 8(4): 351-7.

[20] Darnall B, Ephraim P, Wegener S, et al. Depressive symptoms and mental health service utilization among persons with limb loss: results of a national survey. Arch Phys Med Rehabil 2005; 86(4): 650-8.

[21] Gallagher P, Machlachlan M. Psychological adjustment and coping in adults with prosthetic limbs. Behav Med 1999; 25(3): 117.

[22] Desmond D, MacLachlan M. Psychosocial perspectives on postamputation rehabilitation: a review of disease, trauma, and war related literature. Critic Rev Phys Rehabil Med 2004; 16(2): 77-93.

[23] Vaughn F, Wichowski H, Bosworth G. Does preoperative anxiety level predict postoperative pain? AORN J 2007; 85(3): 589-603.

[24] Ciccozzi A, Marinangeli F, Colangeli A, et al. Anxiolysis and postoperative pain in patients undergoing spinal anesthesia for abdominal hysterectomy. Minerva Anestesiol 2007; 73(7-8): 38793.

[25] Gallagher R, McKinley S. Stressors and anxiety in patients undergoing coronary artery bypass surgery. Am J Crit Care 2007; 16(3): 248-57.

[26] Edwards R, Klick B, Buenaver L, et al. Symptoms of distress as prospective predictors of pain-related sciatica treatment outcomes. Pain 2006; 130(1-2): 47-55. 